Rev. Biol. Trop., 47(1-2): 119-121, 1999

www.ucr.ac.cr www.ots.ac.cr www.ots.duke.edu

\title{
Comparación de la tasa de crecimiento de Penaeus vannamei (Decapoda: Penaeidae) en cultivos semi-intensivos de invierno y de verano
}

\author{
Juan M. Audelo-Naranjo, Olga O. Zamudio-Armenta y José L. Madero-Pérez \\ Facultad de Ciencias del Mar. UAS. Apdo. Postal 610. Paseo Claussen s/n, Mazatlán, Sinaloa, México. \\ Tel. y fax (69) 82-86-56. jaudelo@facimar.maz.uasnet.mx.
}

Recibido 15-IV-1998. Corregido 3-XII-1998. Aceptado 9-XII-1998

\begin{abstract}
The growth rates of Penaeus vannamei in Sinaloa, Mexico, during winter and summer semi-intensive cultures, were compared using a linear regression model that considered age and accumulated temperatures as independent variables. In the first case, the growth curves slope differed significantly, indicating a slower growth in winter; when using accumulated temperatures there was no difference in growth rates indicating that $P$. vannamei at $12 \mathrm{~g}$ (cocktail size) may be harvested when the accumulated weekly temperature reaches 300 $350^{\circ} \mathrm{C}$, depending on the initial size of the organisms in culture.
\end{abstract}

Key words: Growth, temperature, accumulated temperature, P. vannamei.

El registro periódico de la talla de los organismos en estanques de cultivo permite el cálculo de su tasa de crecimiento que puede ser empleada con fines predictivos utilizando el modelo apropiado. Aunque en los crustáceos el crecimiento es discontinuo, en el caso de los peneidos tropicales se utiliza normalmente el modelo lineal, en vista de su alta frecuencia de mudas. A pesar de la importancia económica de este tipo de estudios, la información sobre los factores que influyen mayoritariamente sobre la tasa de crecimiento de los camarones peneidos en cultivo es escasa (Lester y Pante 1992). En este trabajo se examina el crecimiento de Penaeus vannamei (Boone 1931) en dos ciclos de producción en condiciones estacionales diferentes, se determinan las tasas de incremento de peso y se relacionan al historial térmico de los cultivos.

Con esta finalidad se emplearon los datos recabados en cuatro estanques rústicos de la granja acuícola El Yaqui, Sinaloa, México $\left(25^{\circ} 17^{\prime} \mathrm{N}\right.$ y $\left.108^{\circ} 07^{\prime} \mathrm{W}\right)$ durante dos ciclos de cultivo semi-intensivo, el primero en condiciones de invierno (noviembre de 1993 a marzo de 1994) y el segundo en verano (junio-agosto de 1994). La temperatura y la concentración de oxígeno disuelto se registraron a las 6:00 y 13:00 hr de cada día. Semanalmente se recolectaron muestras con una atarraya de $6.4 \mathrm{~mm}$ de luz de malla y $6 \mathrm{~m}^{2}$ de área, tomando al azar 70 camarones por estanque, que se pesaron conjuntamente en una balanza $( \pm 1.0 \mathrm{~g})$ para obtener el peso húmedo promedio individual. Este dato se empleó para generar dos modelos simples de regresión. Con el primero, se determinó la tasa de crecimiento expresada en $\mathrm{g} / \mathrm{se}$ mana para cada situación estacional. Para el segundo se utilizó como variable independiente la temperatura semanal acumulada, la cual se calculó como la suma progresiva de la temperatura promedio semanal. Los criterios de 
ajuste fueron la comparación del error porcentual absoluto (Roff 1983), además del análisis de los residuos y del coeficiente de correlación. Para comprobar diferencias estacionales de las variables ambientales se empleó una prueba de análisis de varianza y para comparar las pendientes se utilizó el análisis de covarianza, con un nivel de significancia de $\mathrm{a}=0.05$.

Variables ambientales: En el ciclo de invierno, los valores promedio de la temperatura de los cuatro estanques variaron entre $19.0 \mathrm{y}$ $19.2^{\circ} \mathrm{C}$ para los datos de la mañana y entre 22.1 y $22.2^{\circ} \mathrm{C}$ para la tarde. El oxígeno disuelto varió entre 4.9 y $5.2 \mathrm{mg} / \mathrm{l}$ por la mañana y entre 7.7 y $7.9 \mathrm{mg} / \mathrm{l}$ por la tarde. En verano, las temperaturas promedio de la mañana fueron de entre 28.0 y $28.5^{\circ} \mathrm{C}$ y en la tarde de 30.8 a $31.5^{\circ} \mathrm{C}$. El oxígeno varió entre 3.5 y 3.7 y entre 7.2 y $7.6 \mathrm{mg} / \mathrm{l}$ por la mañana y por la tarde, respectivamente. Como era de esperar, se encontraron diferencias significativas entre los valores promedio estacionales de las dos variables.

Crecimiento: Los pesos promedio iniciales registrados en los cuatro estanques fueron de entre 0.98 a $1.95 \mathrm{~g}$ para el ciclo de invierno y de $1.8 \mathrm{a} 3.0 \mathrm{~g}$ en el verano. En el primero los pesos finales variaron entre $10.1 \mathrm{a} 11.0 \mathrm{~g}$, después de 14 semanas y en el segundo, en 12 semanas, se cosecharon organismos de 12.0 a $14.1 \mathrm{~g}$. Las ecuaciones de crecimiento en función de la edad o de la temperatura acumulada no revelaron diferencias significativas en lo que se refiere a los errores residuales, al error porcentual absoluto o a los coeficientes de correlación. El análisis de covarianza del modelo que incorpora la variable tiempo indicó que la tasa de crecimiento menor correspondió al ciclo de invierno (Fig. 1A). Por otro lado, el crecimiento guardó una relación directa y significativa con la temperatura acumulada y no se encontraron diferencias de pendientes entre ciclos (Fig. 1B).

La tasa de crecimiento del primer ciclo (0.69 g/semana) indica un crecimiento lento, en tanto que la del segundo cultivo $(0.98 \mathrm{~g} / \mathrm{se}-$ mana) representa un crecimiento aceptable (Anónimo 1988).
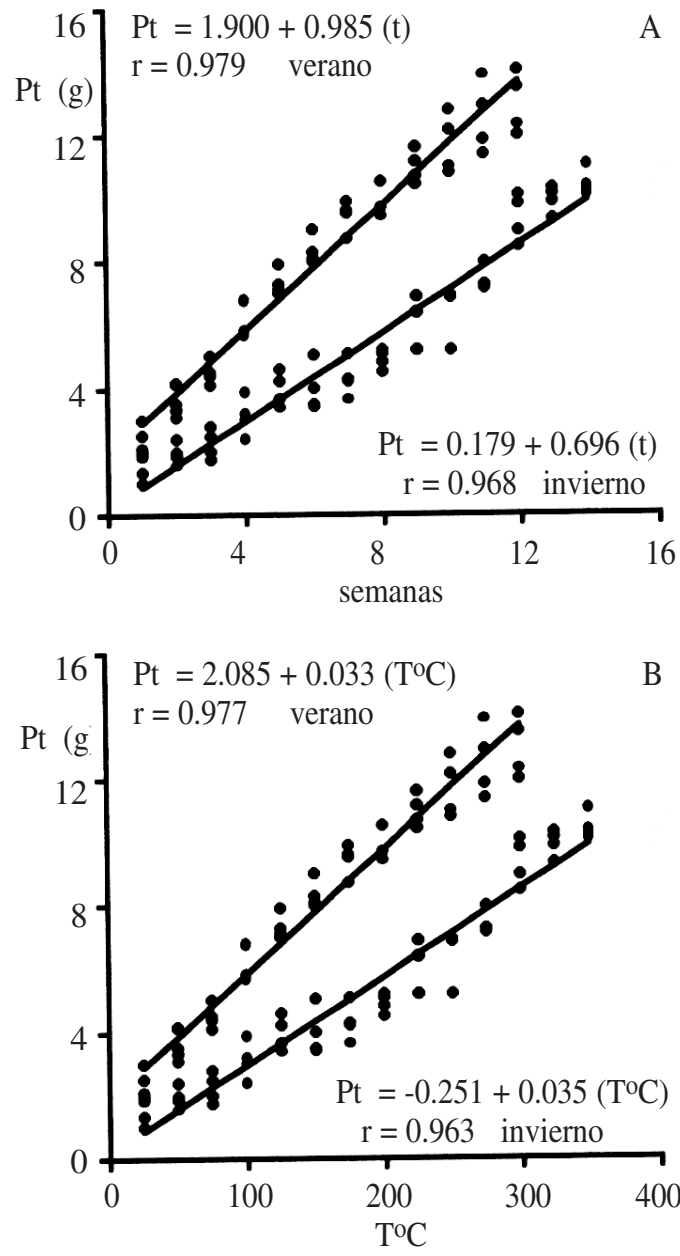

Fig. 1. Curvas de crecimiento de P. vannamei ajustadas en función del tiempo (A) y de la temperatura (B) por ciclo de cultivo.

Considerando que en todos los casos el error porcentual fue menor a $30 \%$, que es el límite aceptable para determinar el valor práctico de un modelo (Roff 1983), que los residuales fueron homogéneos, y que los coeficientes de correlación fueron similares y estadísticamente significativos, puede considerarse que el modelo lineal describe adecuadamente el crecimiento en el tiempo en las dos situaciones estacionales estudiadas.

El crecimiento de un organismo guarda una relación directa con su edad y es bien conocido que cuando no existen factores limitantes, 
como por ejemplo los bajos niveles de oxígeno disuelto que se pueden presentar en la estanquería (Teichert-Coddington 1994), el crecimiento de los peneidos difiere estacionalmente solamente debido al efecto de la temperatura ambiental (Hartnoll 1982). En la granja y en los ciclos objeto de estudio, en los cuales el alimento fue suministrado por demanda y las variables ambientales se mantuvieron dentro de los límites considerados aceptables para el desarrollo de los camarones, la velocidad del crecimiento dependió solamente del tiempo necesario para alcanzar una cantidad aproximadamente similar de grados/semana, independientemente del período del año.

Considerando los pesos iniciales, para alcanzar los $12 \mathrm{~g}$ de peso, que es la talla usual para el mercado nacional, los cultivos de invierno deberían durar 17 semanas para acumular alrededor de $350^{\circ} \mathrm{C}$, mientras que en verano ese tamaño se alcanzaría en 12 semanas, con una temperatura semanal acumulada de cerca de $300^{\circ} \mathrm{C}$; la diferencia en grados acumulados queda explicada por la diferencia en las tallas iniciales de los organismos sembrados en los dos ciclos.

\section{AGRADECIMIENTOS}

Agradecemos a José Calderón Pérez, Domenico Voltolina L. y Aurelio Garibaldi por las oportunas observaciones y sugerencias.

\section{REFERENCIAS}

Anónimo. 1988. Manual de engorda de camarón, cultivo semiintensivo del camarón blanco del Pacífico Mexicano. Fondepesca, México: 101 p.

Hartnoll, R. G. 1982. Growth, p. 111-196. In L. G. Abele (ed.). The Biology of Crustacea, 2. Embryology, Morphology and Genetics. Academic, Nueva York.

Lester, L. J. \& Ma. J. R. Pante. 1992. Penaeid temperature and salinity responses, p. 515-534. In A. W. Fast y L. J. Lester (eds.). Marine Shrimp Culture: Principles and Practices. Developments in Aquaculture and Fisheries Science, 23. Elsevier, Nueva York.

Roff, D. A. 1983. Analysis of catch/effort data a comparison of three methods. Can. J. Fish. Aquat. Sci. 40:1496-1506.

Teichert-Coddington, D. 1994. Seminario Internacional de Camaronicultura en México. R. Purina Internacional, Mazatlán, México. 140 p. 